Supporting Information

\title{
Crystalline Multi-Metal Nanosheets Array with Enriched Oxygen Vacancies as Efficient and Stable Bifunctional Electrocatalysts for Water Splitting
}

Muhammad Imran Abdullah, ${ }^{[c]}$ Asima Hameed, ${ }^{[c]}$ Tuoping Hu, ${ }^{[a]}$ Ning Zhang, ${ }^{*[b]}$ and Mingming $\mathrm{Ma}^{*}[\mathrm{a}, \mathrm{c}]$

Supporting information for this article is given via a link at the end of the document

[a] Department of Chemistry, College of Science, North University of China, Taiyuan 030051, China

[b] Department of Biological and Environmental Engineering, Hefei University, Hefei, Anhui 230022, China

[c] CAS Key Laboratory of Soft Matter Chemistry, Hefei National Laboratory for Physical Sciences at Microscale,

University of Science and Technology of China, Hefei, Anhui 230026, China

E-mail: mma@ustc.edu.cn, zhn@hfuu.edu.cn 


\section{Catalog}

Figure S1. Brunauer-Emmett-Teller (BET) Analysis.

Figure S2. XPS spectrum for CoFeNiO-NSA sample.

Figure S3. XPS spectrum of CoFeNi-NSA sample.

Figure S4. Cyclic voltammograms recorded for the CoFeNiO-NSA.

Figure S5. Cyclic voltammograms recorded for the CoFeNi-NSA.

Table S1. Comparison of the HER performance of non-noble metal based electrocatalysts.

Table S2. Comparison of the OER performance of non-noble metal based electrocatalysts.

Table S3. Comparison of the overall water splitting performance of non-noble metal based electrocatalysts.

Table S4. The amount of elements in a CoFeNiO-NSA.

Table S5. The amount of elements in a CoFeNi-NSA. 


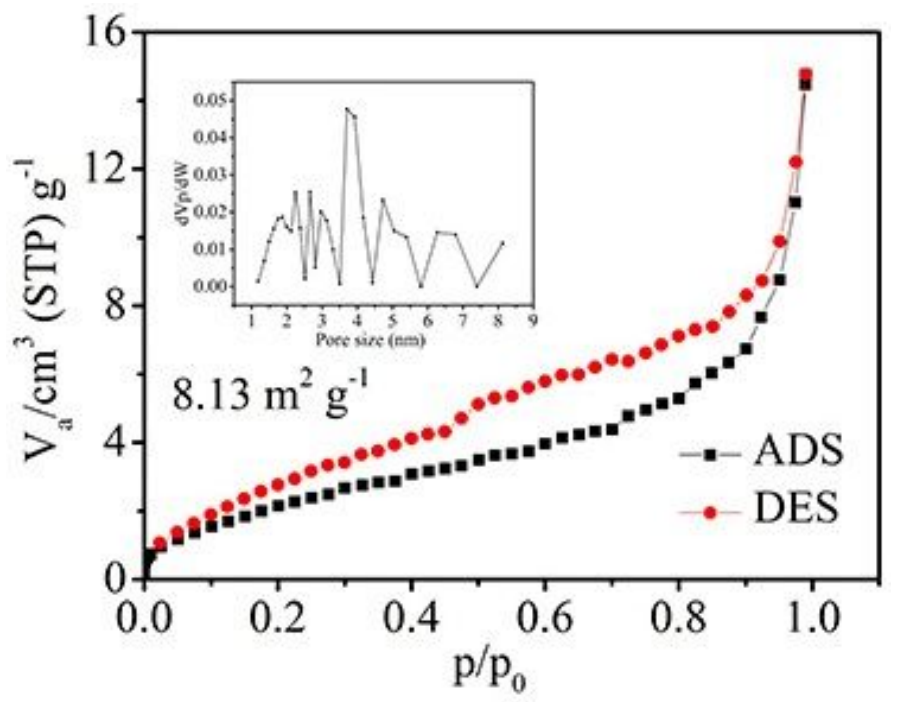

Figure S1. Brunauer-Emmett-Teller (BET) Surface Area Analysis of CoFeNiSe Precursor.

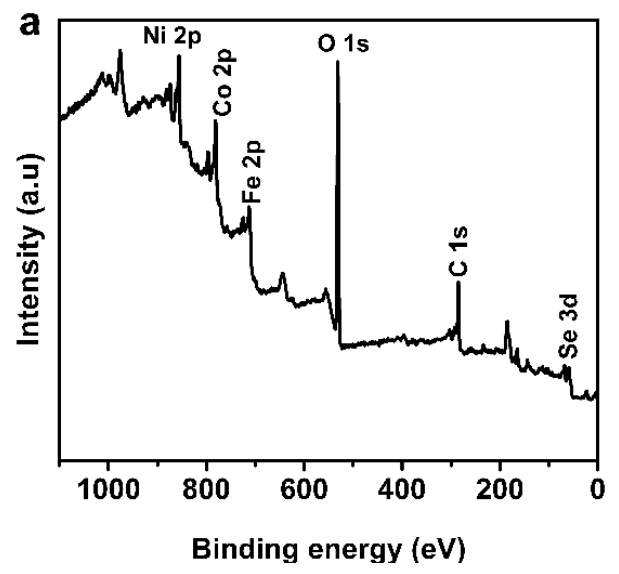

b

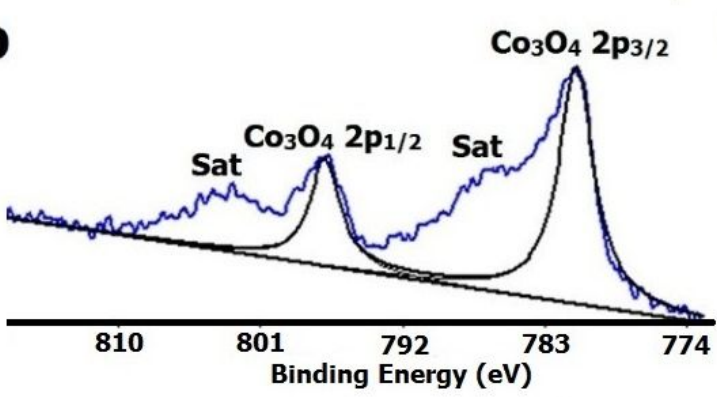

C

d

$\mathrm{NiOOH} \mathrm{2p_{3/2 }}$
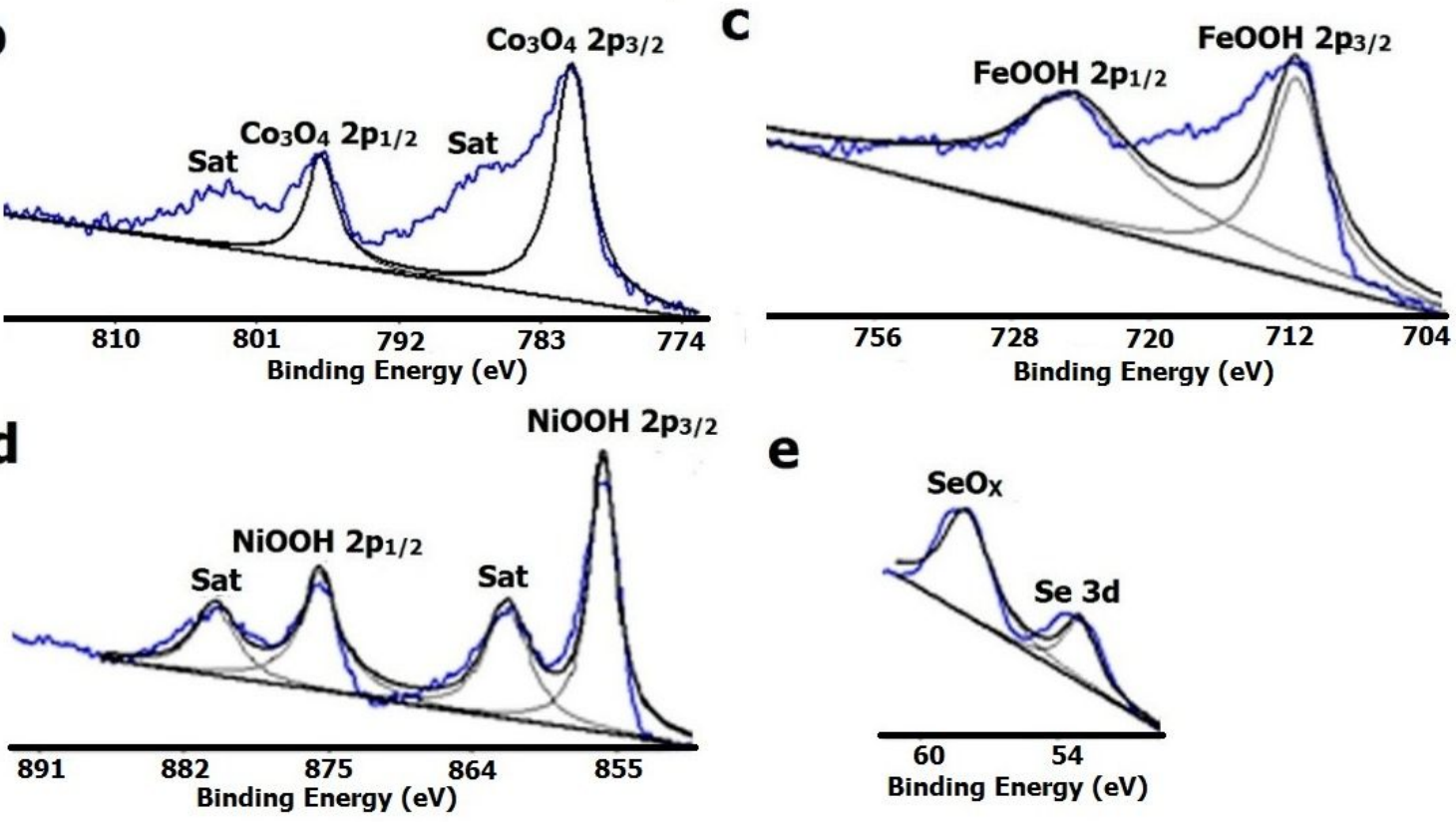

e

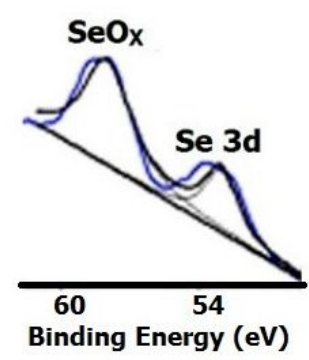

Figure S2. (a)XPS survey spectrum for CoFeNiO-NSA; Core level of XPS spectrum in the (b) Co 2p, (c) Fe 2p, (d) Ni 2p and (e) Se 3d for CoFeNiO-NSA. 


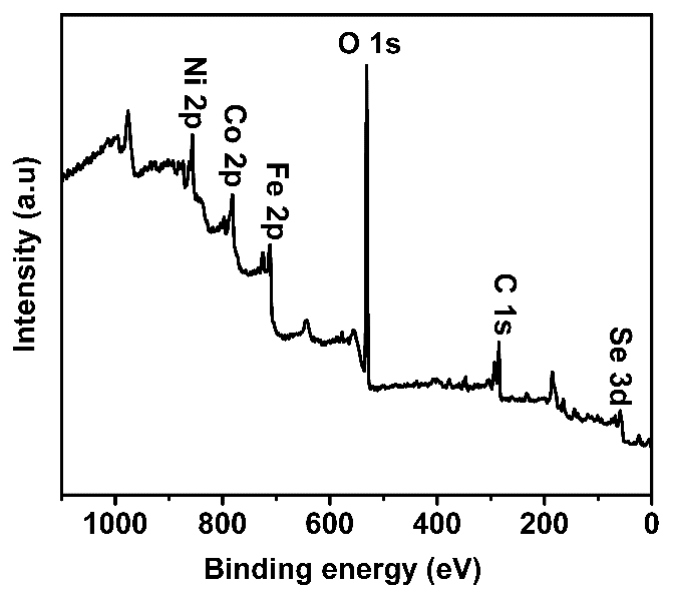

b

c
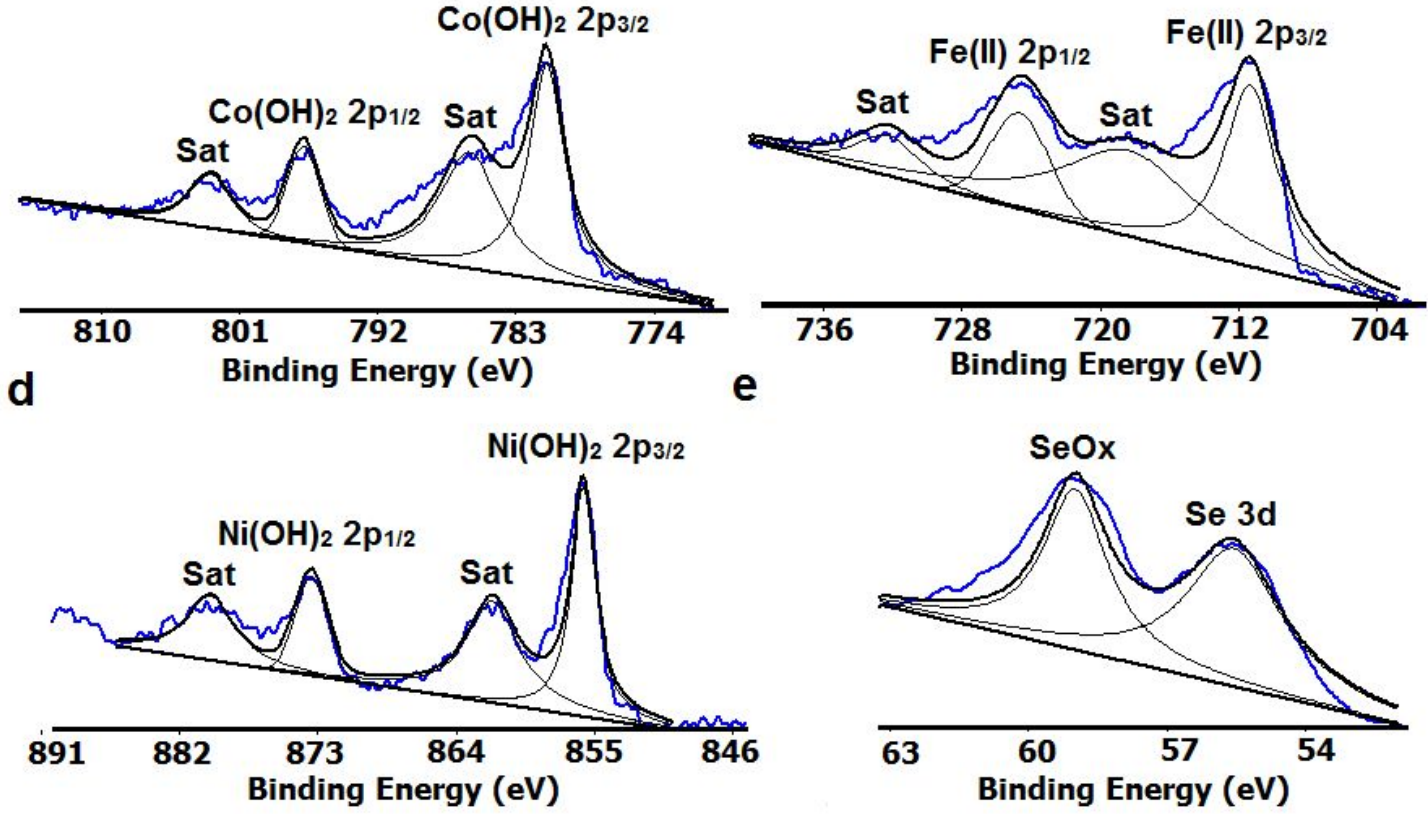

Figure S3 (a)XPS survey spectrum for CoFeNi-NSA; Core level of XPS spectrum in the (b) Co 2p, (c) Fe 2p, (d) Ni 2p and (e) Se 3d for CoFeNi-NSA. 

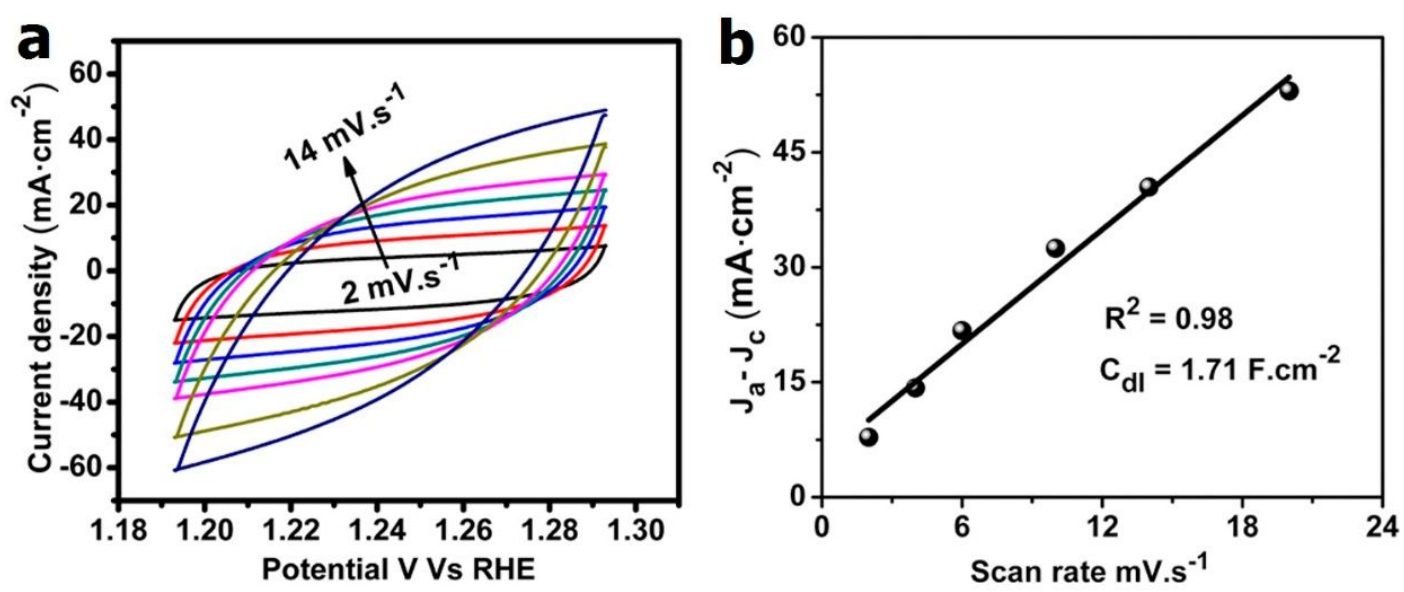

Figure S4 (a) Cyclic voltammograms recorded for the CoFeNiO-NSA electrode at various scan rates in the non-Faradaic region in $1.0 \mathrm{M} \mathrm{KOH}$. (b) Scan rate dependence of the current densities of the CoFeNiO-NSA electrode at $1.24 \mathrm{~V}$ vs RHE. The slope of the lines in (b) is the effective electrochemically active surface area of CoFeNiO-NSA electrodes.
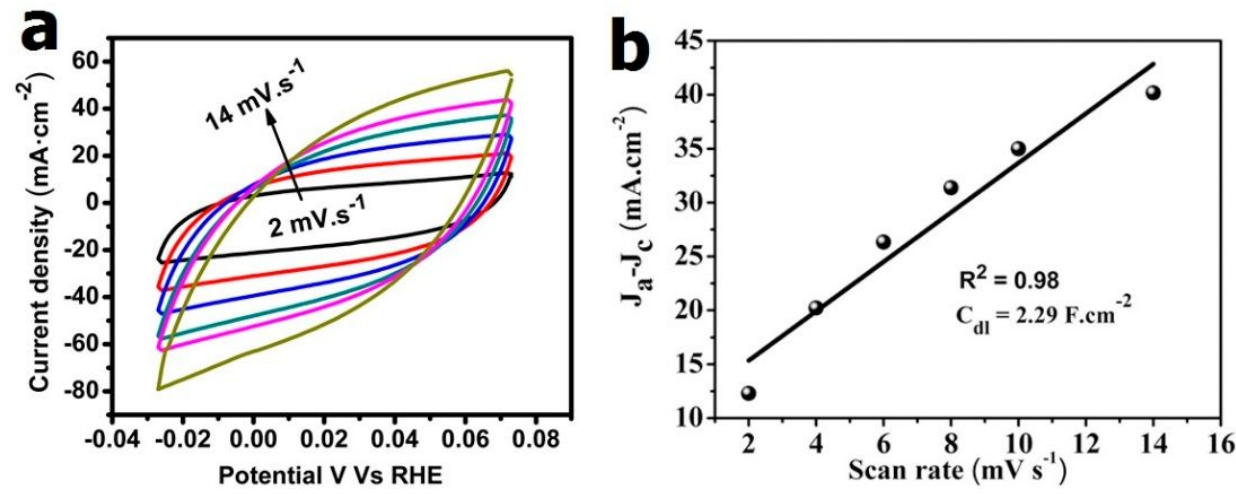

Figure S5 (a) Cyclic voltammograms recorded for the CoFeNi-NSA electrode at various scan rates in the non-Faradaic region in $1.0 \mathrm{M} \mathrm{KOH}$. (b) Scan rate dependence of the current densities of the CoFeNi-NSA electrode at $0.02 \mathrm{~V}$ vs RHE. The slope of the lines in (b) is the effective electrochemically active surface area of CoFeNi-NSA electrodes. 
Table S1. Comparison of CoNiFe-HNP and other non-noble metal electrocatalysts for HER in $1 \mathrm{M} \mathrm{KOH}$.

\begin{tabular}{|c|c|c|c|c|c|}
\hline Material & $\begin{array}{l}\text { Overpotential } \\
\eta(V) \\
\text { @corresponding } \\
\mathrm{j}\end{array}$ & $\begin{array}{c}\text { Current } \\
\text { density } \\
\mathrm{j}(\mathrm{mA} \cdot \mathrm{cm}-2)\end{array}$ & $\begin{array}{l}\text { Tafel slope } \\
\text { (mV-dec-1) }\end{array}$ & $\begin{array}{c}\text { Preparation } \\
\text { method }\end{array}$ & Reference \\
\hline MoB & 0.240 & 20 & 59 & Commercial & 1 \\
\hline CoP nanowire & 0.209 & 10 & 129 & Hydrothermal & 2 \\
\hline Co-NRCNTs & 0.37 & 10 & 69 & Hydrothermal & 3 \\
\hline MoP nanoparticles & 0.13 & 10 & 48 & Sintering & 4 \\
\hline NiO/Ni@CNT & 0.8 & 10 & 82 & Anneanling & 5 \\
\hline MoC & 0.151 & 10 & 59 & Anneanling & 6 \\
\hline MoxC-Ni@NCV & 0.126 & 10 & 93 & Thermolysis & 7 \\
\hline CoOx@CN & 0.232 & 10 & 115 & Hydrothermal & 8 \\
\hline Ni-MoS2 & 0.98 & 10 & 60 & Pyrolysis & 9 \\
\hline Co1Mn1CH/NF & 0.180 & 10 & 60 & Hyrothermal & 10 \\
\hline $\mathrm{NiS}-\mathrm{Ni}_{2} \mathrm{P}_{2} \mathrm{~S}_{6}$ & 0.140 & 10 & 72.8 & Calcination & 11 \\
\hline $\begin{array}{l}\text { CuNDs } / \mathrm{Ni}_{3} \mathrm{~S}_{2} \mathrm{NTs} \\
\text { CFs }\end{array}$ & 0.128 & 10 & 76.2 & Electrodeposition & 12 \\
\hline $\mathrm{Ni}_{2} \mathrm{P} \mathrm{NPs} / \mathrm{C}$ & 0.073 & 10 & 73 & Photochemical & 13 \\
\hline CoNiP-NCP & 0.058 & 10 & 57 & Anneanling & 14 \\
\hline \multirow[t]{3}{*}{ CoFeNi-NSA } & 0.049 & 10 & \multirow[t]{3}{*}{57} & \multirow[t]{3}{*}{ Eletcrodeposition } & \multirow{3}{*}{ This work } \\
\hline & 0.145 & 100 & & & \\
\hline & 0.175 & 200 & & & \\
\hline
\end{tabular}


Table S2. Comparison of CoNiFeO-HNP and other non-noble metal electrocatalysts for OER in $1.0 \mathrm{M} \mathrm{KOH}$

\begin{tabular}{|c|c|c|c|c|c|}
\hline Material & $\begin{array}{l}\text { Overpotential n(V) } \\
\text { @corresponding j }\end{array}$ & $\begin{array}{c}\text { Current } \\
\text { density } \\
\mathrm{j}(\mathrm{mA} \cdot \mathrm{cm}-2)\end{array}$ & $\begin{array}{l}\text { Tafel slope } \\
\text { (mV-dec-1) }\end{array}$ & $\begin{array}{l}\text { Preparation } \\
\text { Methods }\end{array}$ & Reference \\
\hline $\mathrm{Co}_{3} \mathrm{O}_{4} \mathrm{NWS}$ & 0.42 & 20 & 72 & Hydrothermal & 15 \\
\hline $\mathrm{\gamma}-\mathrm{CoOOH}$ & 0.3 & 10 & 38 & Hydrothermal & 16 \\
\hline Co-Fe-P & 0.217 & 10 & 40 & Solvothermal & 17 \\
\hline $\mathrm{Fe} 0.1 \mathrm{Ni0} .9 \mathrm{O}$ & 0.297 & 10 & 37 & Solvothermal & 18 \\
\hline $\mathrm{NiFe} / \mathrm{NF}$ & 0.270 & 10 & 28 & Electrodeposition & 19 \\
\hline $\mathrm{CO}_{4} \mathrm{~N}$ & 0.257 & 10 & 44 & Hydrothermal & 20 \\
\hline $\mathrm{Ni3S2/NF}$ & 0.260 & 10 & 93 & Hydrothermal & 21 \\
\hline CoP nanorods & 0.290 & 10 & 65 & Electrodeposition & 22 \\
\hline $\mathrm{CoSe}_{2}$ sheets & 0.470 & 10 & 64 & Thermal Process & 23 \\
\hline Co-Bi NS/G & 0.29 & 10 & 53 & $\begin{array}{l}\text { Chemical } \\
\text { Reduction }\end{array}$ & 24 \\
\hline $\mathrm{FeOOH} / \mathrm{Co} / \mathrm{FeOOH}$ & 0.25 & 20 & 32 & Electrodeposition & 25 \\
\hline CoMnP & 0.33 & 10 & 61 & Solvothermal & 26 \\
\hline \multirow[t]{3}{*}{ Co-P film } & 0.345 & 10 & \multirow[t]{3}{*}{47} & \multirow[t]{3}{*}{ Electrodeposition } & \multirow[t]{3}{*}{27} \\
\hline & 0.413 & 100 & & & \\
\hline & 0.463 & 500 & & & \\
\hline \multirow{3}{*}{ h-NiSx } & 0.180 & 10 & \multirow[t]{3}{*}{96} & \multirow{3}{*}{$\begin{array}{c}\text { Electrodeposition } \\
\text { Low } \\
\text { Temperature } \\
\text { sulfurization }\end{array}$} & \multirow[t]{3}{*}{28} \\
\hline & 0.217 & 100 & & & \\
\hline & 0.316 & 500 & & & \\
\hline \multirow[t]{3}{*}{ CoFeBO } & 0.240 & 10 & \multirow[t]{3}{*}{53} & \multirow[t]{3}{*}{ Electrodeposition } & \multirow[t]{3}{*}{29} \\
\hline & 0.280 & 100 & & & \\
\hline & 0.310 & 500 & & & \\
\hline \multirow[t]{2}{*}{ G-CoFeW (without iR) } & 0.191 & 10 & \multirow[t]{2}{*}{37} & \multirow[t]{2}{*}{ Sol-gel hyrolysis } & \multirow[t]{2}{*}{30} \\
\hline & 0.260 & 100 & & & \\
\hline CoNiFeB & 0.225 & 10 & 40 & $\begin{array}{c}\text { Chemical } \\
\text { redduction } \\
\text { phosphorization }\end{array}$ & 31 \\
\hline CoFeMo & 0.277 & 10 & 27.74 & solvothermal & 32 \\
\hline $\mathrm{NiS}-\mathrm{Ni}_{2} \mathrm{P}_{2} \mathrm{~S}_{6}$ & 0.220 & 10 & 83.2 & calcination & 11 \\
\hline MoFe:NiOOH/Ni(OH $)_{2}$ & 0.280 & 100 & 47 & Hydrothermal & 33 \\
\hline Fe-Co-P & 0.252 & 10 & 33 & Hydrothermal & 34 \\
\hline
\end{tabular}




\begin{tabular}{|l|l|l|l|l|l|}
\hline P-Co3O4/NF & 0.260 & 20 & 60 & Annealing & 35 \\
\hline CS-NiFeCu & 0.180 & 10 & 33 & Electrodeposition & 36 \\
\hline CoFeNiO-NSA & 0.104 & 10 & 44 & Eletcrodeposition & This Work \\
\cline { 2 - 6 } & 0.240 & 100 & & & \\
\hline
\end{tabular}


Table S3. Comparison of CoNiFeO-HNP II CoNiFe-HNP and other non-noble metal electrocatalysts for overall water splitting in $1.0 \mathrm{M} \mathrm{KOH}$.

\begin{tabular}{|c|c|c|c|c|}
\hline Material & Voltage (V) & $\begin{array}{l}\text { Current density } \\
\mathrm{j}(\mathrm{mA} \cdot \mathrm{cm}-2)\end{array}$ & $\begin{array}{l}\text { Preparation } \\
\text { Methods }\end{array}$ & Reference \\
\hline NiSe NWs $\|$ NiSe NWs & 1.63 & 10 & Hydrothermal & 37 \\
\hline Ni5P4 || Ni5P4 & $\sim 1.70$ & 10 & Hydrothermal & 38 \\
\hline CoP \| CoP & 1.74 & 100 & Electrodeposition & 39 \\
\hline CoP-MNA || CoP-MNA & 1.62 & 10 & Electrodeposition & 40 \\
\hline \multirow[t]{2}{*}{ CoMnO@CN || CoMnO@CN } & 1.7 & 54 & \multirow{2}{*}{$\begin{array}{l}\text { Hydrothermal } \\
\text { Calcination }\end{array}$} & \multirow[t]{2}{*}{41} \\
\hline & 1.8 & 108 & & \\
\hline \multirow[t]{2}{*}{ NiFeOx $\|$ NiFeOx } & 1.7 & 30 & \multirow[t]{2}{*}{ Hydrothermal } & \multirow[t]{2}{*}{42} \\
\hline & 1.8 & 100 & & \\
\hline \multirow{2}{*}{$\begin{array}{l}\mathrm{EG} / \mathrm{Co}_{0.85} \mathrm{Se} / \mathrm{NiFe}-\mathrm{LDH} \| \\
\mathrm{EG} / \mathrm{Co}_{0.85} \mathrm{Se} / \mathrm{NiFe}-\mathrm{LDH}\end{array}$} & 1.67 & 10 & \multirow[t]{2}{*}{ Hydrothermal } & \multirow[t]{2}{*}{43} \\
\hline & 1.71 & 20 & & \\
\hline NiFeS $\|$ NiFeS & 1.62 & 10 & Hydrothermal & 44 \\
\hline $\mathrm{CoMnCH} \| \mathrm{CoMnCH}$ & 1.68 & 10 & Hydrothermal & 10 \\
\hline AlCoP \| AlCoP & 1.56 & 10 & Hydrothermal & 45 \\
\hline $\mathrm{NiS}-\mathrm{Ni}_{2} \mathrm{P}_{2} \mathrm{~S}_{6} \| \mathrm{NiS}-\mathrm{Ni}_{2} \mathrm{P}_{2} \mathrm{~S}_{6}$ & 1.64 & 10 & Calcination & 11 \\
\hline CoFeBO NS \| Co NS & 1.50 & 10 & Eletcrodeposition & 29 \\
\hline $\mathrm{P}_{8.6}-\mathrm{CO}_{3} \mathrm{O}_{4} \| \mathrm{P}_{8.6}-\mathrm{Co}_{3} \mathrm{O}_{4}$ & 1.63 & 10 & Annealing & 35 \\
\hline CoP/NCNHP || CoP/NCNHP & 1.64 & 10 & Pyrolysis & 46 \\
\hline $\mathrm{CO}_{3} \mathrm{O}_{4}$ UNA $\| \mathrm{Co}(\mathrm{OH})_{2}$ UNA & 1.69 & 50 & Eletcrodeposition & 47 \\
\hline \multirow[t]{3}{*}{ CoFeNiO-NSA || CoFeNi-NSA } & 1.39 & 10 & \multirow[t]{3}{*}{ Eletcrodeposition } & \multirow[t]{3}{*}{ This work } \\
\hline & 1.61 & 50 & & \\
\hline & 1.69 & 100 & & \\
\hline
\end{tabular}


Table S4. The amount of elements in a CoFeNiO-NSA (in: \%Atomic)

\begin{tabular}{|c|c|c|c|c|c|}
\hline & Co & $\mathrm{Ni}$ & $\mathrm{Fe}$ & $\mathrm{Se}$ & Co:Ni:Fe:Se \\
\hline $\begin{array}{c}\text { COFeNiO-NSA } \\
\text { before OER }\end{array}$ & 0.1072 & 0.0996 & 0.0870 & 0.1418 & $1.23: 1.14: 1: 1.63$ \\
\hline post-OER & 0.0203 & 0.0245 & 0.0206 & 0.0089 & $1: 1.19: 1: 0.43$ \\
\hline
\end{tabular}

Table S5. The amount of elements in a CoFeNi-NSA (in: \%Atomic)

\begin{tabular}{|c|c|c|c|c|c|}
\hline & Co & $\mathrm{Ni}$ & $\mathrm{Fe}$ & $\mathrm{Se}$ & Co:Ni:Fe:Se \\
\hline CoFeNi-NSA & 0.0810 & 0.1058 & 0.0747 & 0.1401 & $1.08: 1.41: 1: 1.87$ \\
\hline post-HER & 0.0645 & 0.0841 & 0.0644 & 0.0081 & $1: 1.30: 1: 0.12$ \\
\hline
\end{tabular}

\section{References}

1. Vrubel, H.; Hu, X., Molybdenum Boride and Carbide Catalyze Hydrogen Evolution in both Acidic and Basic Solutions. Angew. Chem. Int. Ed. 2012, 51 (51), 12703-12706.

2. Tian, J.; Liu, Q.; Asiri, A. M.; Sun, X., Self-Supported Nanoporous Cobalt Phosphide Nanowire Arrays: An Efficient 3D Hydrogen-Evolving Cathode over the Wide Range of pH 0-14. J. Am. Chem. Soc. 2014, 136 (21), 7587-7590.

3. Zou, X.; Huang, X.; Goswami, A.; Silva, R.; Sathe, B. R.; Mikmeková, E.; Asefa, T., Cobalt-Embedded Nitrogen-Rich Carbon Nanotubes Efficiently Catalyze Hydrogen Evolution Reaction at All pH Values. Angew. Chem. Int. Ed. 2014, 53 (17), 4372-4376.

4. Xiao, P.; Sk, M. A.; Thia, L.; Ge, X.; Lim, R. J.; Wang, J.-Y.; Lim, K. H.; Wang, X., Molybdenum phosphide as an efficient electrocatalyst for the hydrogen evolution reaction. Energy Environ. Sci. 2014, 7 (8), 2624-2629.

5. Gong, M.; Zhou, W.; Tsai, M.-C.; Zhou, J.; Guan, M.; Lin, M.-C.; Zhang, B.; Hu, Y.; Wang, D.-Y.; Yang, J.; Pennycook, S. J.; Hwang, B.-J.; Dai, H., Nanoscale nickel oxide/nickel heterostructures for active hydrogen evolution electrocatalysis. Nat. Commun. 2014, 5 (1), 4695.

6. Wu, H. B.; Xia, B. Y.; Yu, L.; Yu, X.-Y.; Lou, X. W., Porous molybdenum carbide nano-octahedrons synthesized via confined carburization in metal-organic frameworks for efficient hydrogen production. Nat. Commun. 2015, 6 (1), 6512.

7. Wang, S.; Wang, J.; Zhu, M.; Bao, X.; Xiao, B.; Su, D.; Li, H.; Wang, Y., Molybdenum-Carbide-Modified Nitrogen-Doped Carbon Vesicle Encapsulating Nickel Nanoparticles: A Highly Efficient, Low-Cost Catalyst for Hydrogen Evolution 
Reaction. J. Am. Chem. Soc. 2015, 137 (50), 15753-15759.

8. Jin, H.; Wang, J.; Su, D.; Wei, Z.; Pang, Z.; Wang, Y., In situ CobaltCobalt Oxide/N-Doped Carbon Hybrids As Superior Bifunctional Electrocatalysts for Hydrogen and Oxygen Evolution. J. Am. Chem. Soc. 2015, 137 (7), 2688-2694.

9. Ma, Y.-Y.; Wu, C.-X.; Feng, X.-J.; Tan, H.-Q.; Yan, L.-K.; Liu, Y.; Kang, Z.-H.; Wang, E.-B.; Li, Y.-G., Highly efficient hydrogen evolution from seawater by a low-cost and stable CoMoP@C electrocatalyst superior to Pt/C. Energy Environ. Sci. 2017, 10 (3), 788-798.

10. Tang, T.; Jiang, W.-J.; Niu, S.; Liu, N.; Luo, H.; Chen, Y.-Y.; Jin, S.F.; Gao, F.; Wan, L.-J.; Hu, J.-S., Electronic and Morphological Dual Modulation of Cobalt Carbonate Hydroxides by Mn Doping toward Highly Efficient and Stable Bifunctional Electrocatalysts for Overall Water Splitting. J. Am. Chem. Soc. 2017, 139 (24), 8320-8328.

11. Zhang, X.; Zhang, S.; Li, J.; Wang, E., One-step synthesis of well-structured NiS-Ni2P2S6 nanosheets on nickel foam for efficient overall water splitting. $J$. Mater. Chem. A 2017, 5 (42), 22131-22136.

12. Feng, J.-X.; Wu, J.-Q.; Tong, Y.-X.; Li, G.-R., Efficient Hydrogen Evolution on $\mathrm{Cu}$ Nanodots-Decorated Ni3S2 Nanotubes by Optimizing Atomic Hydrogen Adsorption and Desorption. J. Am. Chem. Soc. 2018, 140 (2), 610-617.

13. Lin, Y.; He, L.; Chen, T.; Zhou, D.; Wu, L.; Hou, X.; Zheng, C., Costeffective and environmentally friendly synthesis of $3 \mathrm{D} \mathrm{Ni} \mathrm{Ni}_{2} \mathrm{P}$ from scrap nickel for highly efficient hydrogen evolution in both acidic and alkaline media. J, Mater. Chem. A 2018, 6 (9), 4088-4094.

14. Fang, Z.; Peng, L.; Qian, Y.; Zhang, X.; Xie, Y.; Cha, J. J.; Yu, G., Dual Tuning of $\mathrm{Ni}-\mathrm{Co}-\mathrm{A}(\mathrm{A}=\mathrm{P}, \mathrm{Se}, \mathrm{O})$ Nanosheets by Anion Substitution and Holey Engineering for Efficient Hydrogen Evolution. J. Am. Chem. Soc. 2018, 140 (15), 5241-5247.

15. Wang, Y.; Zhou, T.; Jiang, K.; Da, P.; Peng, Z.; Tang, J.; Kong, B.; Cai, W.-B.; Yang, Z.; Zheng, G., Reduced Mesoporous $\mathrm{Co}_{3} \mathrm{O}_{4}$ Nanowires as Efficient Water Oxidation Electrocatalysts and Supercapacitor Electrodes. Adv. Energy Mater. 2014, 4 (16), 1400696.

16. Huang, J.; Chen, J.; Yao, T.; He, J.; Jiang, S.; Sun, Z.; Liu, Q.; Cheng, W.; Hu, F.; Jiang, Y., CoOOH nanosheets with high mass activity for water oxidation. Angew. Chem. Int. Ed. 2015, 54 (30), 8722-8727.

17. Wang, T.; Wang, C.; Jin, Y.; Sviripa, A.; Liang, J.; Han, J.; Huang, Y.; Li, Q.; Wu, G., Amorphous Co-Fe-P nanospheres for efficient water oxidation. J. Mater. Chem. A 2017, 5 (48), 25378-25384.

18. Fominykh, K.; Chernev, P.; Zaharieva, I.; Sicklinger, J.; Stefanic, G.; Döblinger, M.; Müller, A.; Pokharel, A.; Böcklein, S.; Scheu, C., Iron-doped nickel oxide nanocrystals as highly efficient electrocatalysts for alkaline water splitting. ACS Nano 2015, 9 (5), 5180-5188.

19. Lu, X.; Zhao, C., Electrodeposition of hierarchically structured three-dimensional nickel-iron electrodes for efficient oxygen evolution at high current densities. Nat.

Commun. 2015, 6, 6616. 
20. Chen, P.; Xu, K.; Fang, Z.; Tong, Y.; Wu, J.; Lu, X.; Peng, X.; Ding, H.; Wu, C.; Xie, Y., Metallic $\mathrm{Co}_{4} \mathrm{~N}$ porous nanowire arrays activated by surface oxidation as electrocatalysts for the oxygen evolution reaction. Angew. Chem. Int. Ed. 2015, 54 (49), 14710-14714.

21. Feng, L.-L.; Yu, G.; Wu, Y.; Li, G.-D.; Li, H.; Sun, Y.; Asefa, T.; Chen, W.; Zou, X., High-index faceted $\mathrm{Ni}_{3} \mathrm{~S}_{2}$ nanosheet arrays as highly active and ultrastable electrocatalysts for water splitting. J. Am. Chem. Soc. 2015, 137 (44), 14023-14026.

22. Zhu, Y. P.; Liu, Y. P.; Ren, T. Z.; Yuan, Z. Y., Self-supported cobalt phosphide mesoporous nanorod arrays: a flexible and bifunctional electrode for highly active electrocatalytic water reduction and oxidation. Adv. Funct. Mater. 2015, 25 (47), 7337-7347.

23. Liang, L.; Cheng, H.; Lei, F.; Han, J.; Gao, S.; Wang, C.; Sun, Y.; Qamar, S.; Wei, S.; Xie, Y., Metallic Single - Unit - Cell Orthorhombic Cobalt Diselenide Atomic Layers: Robust Water - Electrolysis Catalysts. Angew. Chem. Int. Ed. 2015, 54 (41), 12004-12008.

24. Chen, P.; Xu, K.; Zhou, T.; Tong, Y.; Wu, J.; Cheng, H.; Lu, X.;

Ding, H.; Wu, C.; Xie, Y., Strong-coupled cobalt borate nanosheets/graphene hybrid as electrocatalyst for water oxidation under both alkaline and neutral conditions.

Angew. Chem. Int. Ed. 2016, 55 (7), 2488-2492.

25. Feng, J. X.; Xu, H.; Dong, Y. T.; Ye, S. H.; Tong, Y. X.; Li, G. R., $\mathrm{FeOOH} / \mathrm{Co} / \mathrm{FeOOH}$ hybrid nanotube arrays as high - performance electrocatalysts for the oxygen evolution reaction. Angew. Chem. Int. Ed. 2016, 55 (11), 3694-3698.

26. Li, D.; Baydoun, H.; Verani, C. u. N.; Brock, S. L., Efficient water oxidation using CoMnP nanoparticles. J. Am. Chem. Soc. 2016, 138 (12), 4006-4009.

27. Jiang, N.; You, B.; Sheng, M.; Sun, Y., Electrodeposited cobalt-phosphorousderived films as competent bifunctional catalysts for overall water splitting. Angew.

Chem. Int. Ed. 2015, 54 (21), 6251-6254.

28. You, B.; Sun, Y., Hierarchically porous nickel sulfide multifunctional superstructures. Adv. Energy Mater. 2016, 6 (7), 1502333.

29. Liu, B.; Zhang, N.; Ma, M., Cobalt-based nanosheet arrays as efficient electrocatalysts for overall water splitting. J. Mater. Chem. A 2017, 5 (33), 1764017646.

30. Zhang, B.; Zheng, X.; Voznyy, O.; Comin, R.; Bajdich, M.; GarcíaMelchor, M.; Han, L.; Xu, J.; Liu, M.; Zheng, L., Homogeneously dispersed multimetal oxygen-evolving catalysts. Science 2016, 352 (6283), 333-337.

31. Wu, Z.; Nie, D.; Song, M.; Jiao, T.; Fu, G.; Liu, X., Facile synthesis of Co-Fe-B-P nanochains as an efficient bifunctional electrocatalyst for overall watersplitting. Nanoscale 2019, 11 (15), 7506-7512.

32. Liu, P. F.; Yang, S.; Zheng, L. R.; Zhang, B.; Yang, H. G., Mo 6+ activated multimetal oxygen-evolving catalysts. Chem. Sci. 2017, 8 (5), 3484-3488.

33. Jin, Y.; Huang, S.; Yue, X.; Du, H.; Shen, P. K., Mo- and Fe-Modified $\mathrm{Ni}(\mathrm{OH})_{2} / \mathrm{NiOOH}$ Nanosheets as Highly Active and Stable Electrocatalysts for Oxygen Evolution Reaction. ACS Catal. 2018, 8 (3), 2359-2363. 
34. Liu, K.; Zhang, C.; Sun, Y.; Zhang, G.; Shen, X.; Zou, F.; Zhang, H.; Wu, Z.; Wegener, E. C.; Taubert, C. J.; Miller, J. T.; Peng, Z.; Zhu, Y., HighPerformance Transition Metal Phosphide Alloy Catalyst for Oxygen Evolution Reaction. ACS Nano 2018, 12 (1), 158-167.

35. Wang, Z.; Liu, H.; Ge, R.; Ren, X.; Ren, J.; Yang, D.; Zhang, L.; Sun, X., Phosphorus-Doped $\mathrm{Co}_{3} \mathrm{O}_{4}$ Nanowire Array: A Highly Efficient Bifunctional Electrocatalyst for Overall Water Splitting. ACS Catal. 2018, 8 (3), 2236-2241. 36. Zhang, P.; Li, L.; Nordlund, D.; Chen, H.; Fan, L.; Zhang, B.; Sheng, X.; Daniel, Q.; Sun, L., Dendritic core-shell nickel-iron-copper metal/metal oxide electrode for efficient electrocatalytic water oxidation. Nat. Commun. 2018, 9 (1), 381.

37. Tang, C.; Cheng, N.; Pu, Z.; Xing, W.; Sun, X., NiSe Nanowire Film Supported on Nickel Foam: An Efficient and Stable 3D Bifunctional Electrode for Full Water Splitting. Angew. Chem. Int. Ed. 2015, 54 (32), 9351-9355.

38. Ledendecker, M.; Krick Calderón, S.; Papp, C.; Steinrück, H.-P.; Antonietti, M.; Shalom, M., The Synthesis of Nanostructured $\mathrm{Ni}_{5} \mathrm{P}_{4}$ Films and their Use as a Non-Noble Bifunctional Electrocatalyst for Full Water Splitting. Angew. Chem. Int. Ed. 2015, 127 (42), 12538-12542.

39. Jiang, N.; You, B.; Sheng, M.; Sun, Y., Electrodeposited CobaltPhosphorous-Derived Films as Competent Bifunctional Catalysts for Overall Water Splitting. Angew. Chem. Int. Ed. 2015, 54 (21), 6251-6254.

40. Zhu, Y.-P.; Liu, Y.-P.; Ren, T.-Z.; Yuan, Z.-Y., Self-Supported Cobalt Phosphide Mesoporous Nanorod Arrays: A Flexible and Bifunctional Electrode for Highly Active Electrocatalytic Water Reduction and Oxidation. Adv. Funct. Mater. 2015, 25 (47), 7337-7347.

41. Li, J.; Wang, Y.; Zhou, T.; Zhang, H.; Sun, X.; Tang, J.; Zhang, L.; Al-Enizi, A. M.; Yang, Z.; Zheng, G., Nanoparticle Superlattices as Efficient Bifunctional Electrocatalysts for Water Splitting. J. Am. Chem. Soc. 2015, 137 (45), 14305-14312.

42. Wang, H.; Lee, H.-W.; Deng, Y.; Lu, Z.; Hsu, P.-C.; Liu, Y.; Lin, D.; Cui, Y., Bifunctional non-noble metal oxide nanoparticle electrocatalysts through lithium-induced conversion for overall water splitting. Nat. Commun. 2015, 6 (1), 7261.

43. Hou, Y.; Lohe, M. R.; Zhang, J.; Liu, S.; Zhuang, X.; Feng, X., Vertically oriented cobalt selenide/NiFe layered-double-hydroxide nanosheets supported on exfoliated graphene foil: an efficient 3D electrode for overall water splitting. Energy Environ. Sci. 2016, 9 (2), 478-483.

44. Yu, J.; Cheng, G.; Luo, W., Ternary nickel-iron sulfide microflowers as a robust electrocatalyst for bifunctional water splitting. J. Mater. Chem. A 2017, 5 (30), 15838-15844.

45. Zhang, R.; Tang, C.; Kong, R.; Du, G.; Asiri, A. M.; Chen, L.; Sun, X., Al-Doped CoP nanoarray: a durable water-splitting electrocatalyst with superhigh activity. Nanoscale 2017, 9 (14), 4793-4800.

46. Pan, Y.; Sun, K.; Liu, S.; Cao, X.; Wu, K.; Cheong, W.-C.; Chen, Z.; 
Wang, Y.; Li, Y.; Liu, Y.; Wang, D.; Peng, Q.; Chen, C.; Li, Y., Core-Shell ZIF-8@ZIF-67-Derived CoP Nanoparticle-Embedded N-Doped Carbon Nanotube Hollow Polyhedron for Efficient Overall Water Splitting. J. Am. Chem. Soc. 2018, 140 (7), 2610-2618.

47. Zhang, L.; Liu, B.; Zhang, N.; Ma, M., Electrosynthesis of $\mathrm{Co}_{3} \mathrm{O}_{4}$ and $\mathrm{Co}(\mathrm{OH})_{2}$ ultrathin nanosheet arrays for efficient electrocatalytic water splitting in alkaline and neutral media. Nano Res. 2018, 11 (1), 323-333. 